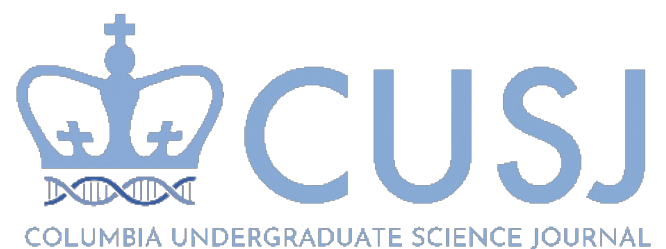

\title{
The Effects of APOBEC3G's Cytidine Deaminase Activity on Retroviral Evolution
}

\author{
Mary-Benedicta Obikili \\ 1Department of Microbiology and Immunology, University of Illinois at Chicago College of Medicine, \\ Chicago, IL, USA
}

KEYWORDS: APOBEC3G, cytidine deamination, retroviral evolution

ABSTRACT: Apolipoprotein B editing complex (APOBEC3/A3) genes are found in mammalian cells. In primates, there are 7 APOBEC3 genes, namely, 3A, 3B, 3C, 3DE, 3F, 3G, and $3 \mathrm{H}$. Previous research has shown that $\mathrm{A} 3$ proteins help to inhibit viral infection via their cytidine deaminase activity. However, it has also been found that A3 proteins could lead to viral evolution, where retroviruses such as HIV (Human Immunodeficiency Virus), acquire beneficial mutations that enable them to overcome the antiviral activity of A3 proteins, gain resistance to certain drugs used for treating viral infections and escape recognition by the immune system. This paper is a review article summarizing the role of $A 3 G$ on viral infection and evolution, and the potential impact viral evolution could have in treatment of retroviral infections such as HIV.

\section{INTRODUCTION}

Apolipoprotein B- Editing Complex 3 (APOBEC3/A3) genes encode enzymes that prevent viral infection through their cytidine deaminase activity [1]. There are seven APOBEC3 genes found in humans, namely: $A 3 A, A 3 B, A 3 C$, A3DE, A3F, A3G, and A3H. Most of these proteins play a role in inhibiting viral infection, however, A3G, A3F, A3D, and A3H are best known for their antiviral role [2, 3]. A3 proteins can be found in human and mouse dendritic and myeloid cells, and they are usually expressed at different levels in hematopoietic cell populations, which include B cells, CD4+ and CD8+ $T$ cells [1]. When a host cell is infected, interferons, and other chemokines and cytokines are released and stimulate the expression of A3 proteins in other cells which help to fight off the infection [1]. It has been found that $A 3$ proteins, specifically $A 3 G$, is the main antiviral protein in CD4+ T cells. Therefore, when a cell is infected, A3G levels increase, hence inhibiting viral replication by introducing more $G$ to $A$ hypermutations in the virus [1]. These A3 proteins are packaged into the virion, which lead to viral inhibition through their cytidine deaminase activity. The cytidine deaminase activity of $\mathrm{A} 3$ proteins refers to the conversion of cytosine to uracil in single-stranded DNA (Fig. 1A). During the process of retrovirus infection, the virion enters the cell cytoplasm and uncoats its viral RNA (Fig. 1B). The viral enzyme reverse transcriptase then uses the RNA as a template for making DNA. However, during the process of reverse transcription, the A3 protein deaminates the cytidine residues of the (-) strand vi-

(C) 2021 Obikili. This is an open access article distributed under the terms of the Creative Commons Attribution License, which permits the user to copy, distribute, and transmit the work provided that the original authors and source are credited. 
A

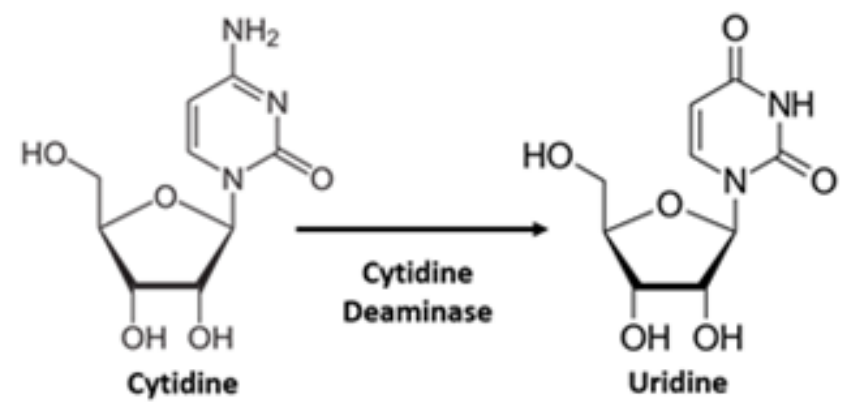

B

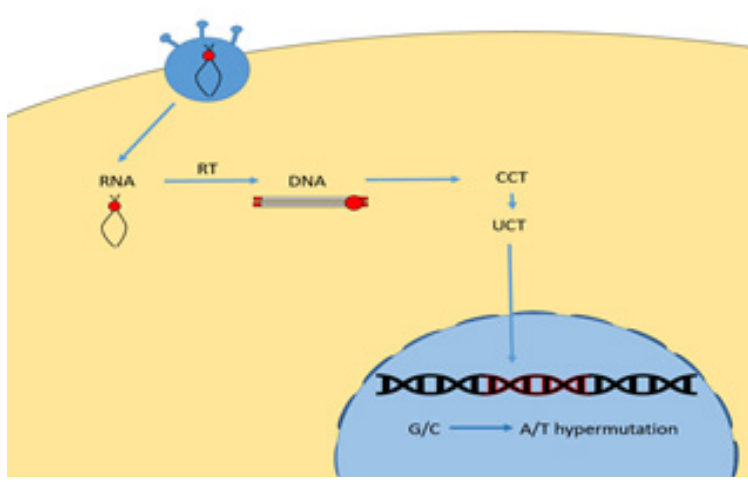

Figure 1: The process of cytidine deamination.

(A) The enzyme APOBEC3, which is a cytidine deaminase, deaminates cytidine to uridine in the single-stranded retroviral DNA before its integration into the host cell

(Source: National University of Singapore, Faculty of Science, Special Programme in Science and iGEM 2018, Yuhui Deborah Fong (used with permission))

(B) The virus enters the cell. After uncoating of the RNA genome, the viral enzyme, reverse transcriptase makes a double-stranded DNA copy of the genome, which then enters the nucleus and integrates into the host chromosomes. During reverse transcription, A3 enzymes (red ball), which are packaged in the virion deaminates cytidine to uracil in the single-stranded viral DNA before its replication and integration into the chromosomes, resulting in G-to-A changes in the viral genome. The red DNA in the nucleus is the integrated viral DNA.

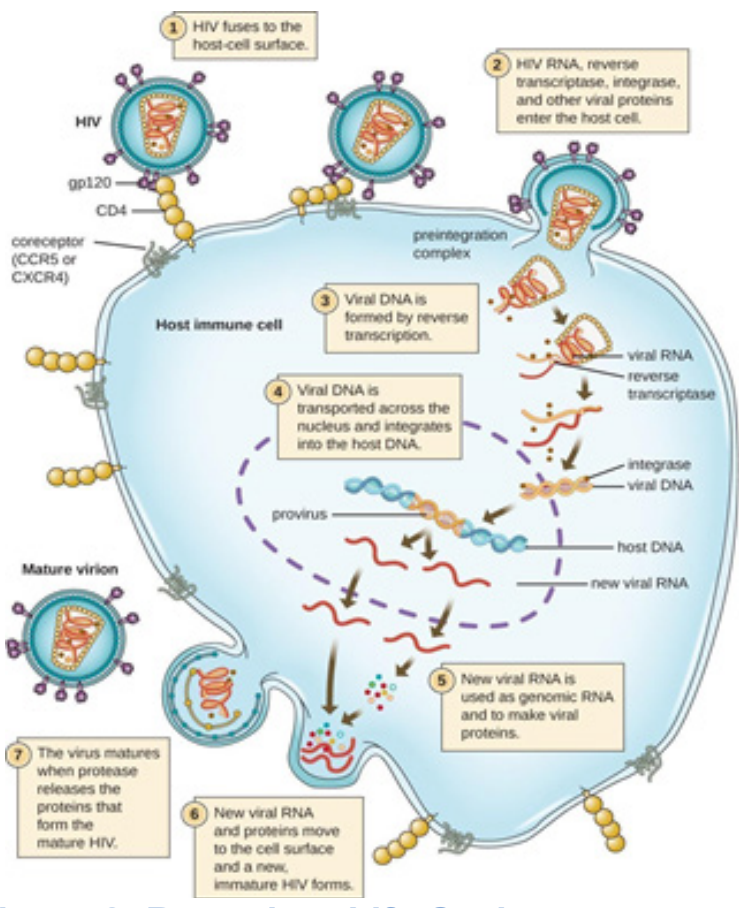

Figure 2: Retrovirus Life Cycle

This shows the process of retroviral integration and replication in the host cell.

(Figure obtained and used with permission from OpenStax Microbiology textbook [7]) (Access at https://openstax.org/books/microbiology/pages/1-introduction) ral DNA to uracil residues, resulting in G-to-A mutations in the viral coding (+) strand DNA. In other words, because cytidine is converted to uracil, which reverse transcriptase reads as thymidine, guanosine is replaced with adenine in the viral coding strand DNA. After the synthesis of a double stranded DNA molecule, the viral DNA goes into the host cell's nucleus and becomes integrated in the chromosomes (Fig. 1B). The A3-induced mutations inhibit the synthesis of virus proteins and virions because they inactivate the virus, which prevents it from infecting and replicating in other cells.

Although APOBEC3 proteins help inhibit viral infection through their cytidine deaminase activity, A3D, A3F, A3G, and A3H may also play a role in viral evolution, where viruses such as HIV gain beneficial mutations that enable them to overcome the antiviral activity of A3 proteins $[5,6]$. This paper will highlight the role of $A 3$ proteins, specifically $A 3 G$, on retroviral evolution.

\section{RETROVIRUS REPLICATION}

Viruses are infectious pathogens that require a host cell for their growth and replication. Al- 
though they do not have the ability to grow on their own, once inside a cell they use its machinery to grow and multiply and have the potential to cause diseases in the host organism.

Retroviruses are viruses that use their viral RNA-dependent enzyme, reverse transcriptase, to synthesize viral DNA in a host cell which is then inserted into the chromosomal DNA (Fig. 1B). HIV is a lentivirus that infects CD4+ T cells, macrophages, and dendritic cells in humans [1]. The process of retrovirus infection is shown in Fig. 2. Once a retrovirus encounters a host cell, it binds to a cell surface receptor, hence enabling its fusion with the cell. After it fuses with the cell, it uncoats its RNA genome and enzymes, such as reverse transcriptase (RT), which then synthesize a new copy of the viral DNA from the RNA template. Then the viral DNA enters the nucleus and the viral enzyme integrase, along with several host proteins, splices the viral DNA into the cell's genome. Cellular RNA polymerase is then used to transcribe the viral DNA into mRNA which is then translated into viral protein. The new virions made are assembled at the host membrane and bud off from the cell membrane.

The final stage consists of the retrovirus protease enzymecleaving the precursorproteins into mature viral proteins leading to the maturation of the virus and the start of a new cycle [8].

\section{RETROVIRAL EVOLUTION}

The cytidine deaminase activity of $\mathrm{A} 3$ proteins may also lead to retroviral evolution, where the virus gains beneficial mutations that help the virus to counteract $A 3$ antiviral activity $[5,6]$. A3G has been shown to preferentially deaminate $\underline{C} C$ motifs, resulting in $\underline{A} G$ motifs in the coding strand, whereas the other A3 proteins have been shown to preferentially deaminate Tㅡㅡ motifs, resulting in A스 motifs [5, 6]. However, HIV-1 encodes a protein the viral infectivity factor (Vif), which leads to the degradation of A3 proteins in virus-producing cells [5]. Vif has two motifs that bind to $A 3$ proteins: DRMR which binds to and degrades $\mathrm{A} 3 \mathrm{~F}$ and $\mathrm{A} 3 \mathrm{D}$, and the other motif, YRHHY, which binds to A3G, leading to its degradation [5]. Fig. 3 shows the structure of the Vif complex [5]. When Vif binds to $A 3$, it prevents it from entering the budding virion by targeting it for degradation. It does this by binding to CBFB co-factor and Cullin 5 E3 Ubiquitin Ligase (ELOB-ELOC-CUL5), via the sites diagrammed in Fig. 3B, which then targets the A3 protein for proteasomal degradation [5].

A
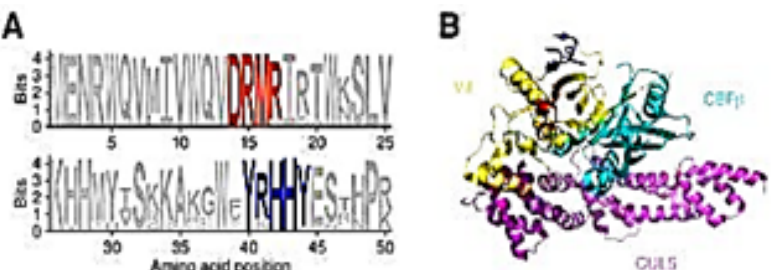

Figure 3: The structure of Vif

(A) Shows the two Vif motifs that bind to A3 proteins. DRMR (red) binds to A3D and A3F, whereas YRHHY (blue) binds to A3G

(B) Shows the Vif complex. The blue and red correspond to the Vif binding motifs in A where Vif binds to A3D, A3F and A3G, respectively. The yellow, cyan and pink colors represent the Vif protein, CBFB co-factor and CUL5 ligase respectively (Fig. obtained from Sato K. et al. 2014, with permission to reprint from PLOS pathogens).

Viral evolution can occur when there are defective (mutant) Vif proteins. The defective proteins do not cause complete A3 degradation and therefore allow some $\mathrm{A} 3$ proteins to enter the virion, resulting in mutations in the viral genome. Since A3G prefers to deaminate TGGG - TAGG motifs, it often converts the codon encoding for the amino acid tryptophan (TGG) into a stop codon (TAG), which renders the virus defective and unable to synthesize its proteins. This prevents it from assembling at the host cell's membrane and infecting other cells. Sato et al. found that A3 proteins lead to mutations in the env region of the HIV-1 genome, which codes for the viral glycoprotein [5]. This protein is important for enabling the virus to bind to cellular receptors and fuse with the host cell's membrane. Mutations caused by 
A3 proteins inhibit the virus from synthesizing its glycoprotein, which in turn prevents it from infecting the cell. These defective viruses may later get degraded. Occasionally, the resulting G-to-A mutations may also be sub-lethal (depending on the levels of $A 3 G$ present in the cell, which may be due to genetic factors, such as different polymorphisms in A3G genes in humans [9], and the context of the TG motif), resulting in missense mutations. In other words, lower levels of A3G expression, would result in more sub-lethal mutations, compared to higher levels of A3G expression, which would mostly result in lethal mutations. Moreover, other $A 3$ proteins such as $A 3 D$ and $A 3 F$, respectively prefer deaminating the $\underline{G} A-\underline{A} A$ and $\underline{G} A A$ - AAA motifs [5], resulting in sub-lethal missense mutations. These missense mutations may result in viral evolution and diversification. For example, the Sato study showed that $A 3$ mutations in env also changed the co-receptor specificity of the glycoprotein, enabling it to preferentially infect different cell types. It has been found that some $\mathrm{A} 3$ proteins may indirectly contribute to retroviral evolution, where the cytidine deaminase activity of the A3 protein helps the virus to acquire mutations that will make it more fit. Studies by Kim et al., Sato et al., and Fourati et al. showed that retroviruses, such as HIV, can sometimes gain beneficial mutations that enable them to replicate better $[3,5,6]$. Sato et al., Fourati et al., and Borzooee et al., also demonstrated that A3 proteins such as A3D, A3F, A3G and A3H can sometimes lead to retroviral evolution [3, $5,10]$. It was also shown that A3 proteins can mutate viruses so that they can use different receptors on the cell surface for entry into the host cell [5]. Moreover, in the Sato study [5], where humanized mice models were used to study different Vif mutants that were initially mutated via A3-independent means, it was found that $A 3 D$ and $A 3 F$ highly suppressed one mutant, A3G highly suppressed another mutant, and the other was a double mutant, that was suppressed by A3D, A3F, and A3G [5]. It was demonstrated that although a wild type Vif protein can target A3 for proteasomal degradation, a defective Vif protein, could be suppressed by A3 proteins. However, sometimes these A3-dependent mutations may benefit the virus. In the study, they found that A3F and A3D led to more viral diversification compared to A3G (for reasons previously described).

These studies suggest that $\mathrm{A} 3$ proteins could benefit the virus by leading to mutations that will help the virus to overcome A3's antiviral activity, and evade antiretroviral drugs detection, leading to viral escape. This will enable them to continue to undergo replication and infection in additional cells. In other words, the A3 proteins assist the virus by enabling them to gain diversity, which in turn may help the virus to acquire mutations that make it gain drug resistance.

\section{DRUG RESISTANCE}

Drug resistance refers to the ability of a pathogen to overcome the effects of a drug. This usually occurs when a certain drug is continuously used to treat a particular infection, and due to constant exposure of the disease pathogen to the drug, the drug becomes less effective, usually because the pathogen acquires drug-resistance gene mutations. To minimize the possibility of disease pathogens gaining resistance, multiple drugs could be used for the treatment of that disease. This is usually seen in the treatment of certain diseases, such as HIV, where a combination of different drugs (such as highly active antiretroviral therapy, HAART) are used simultaneously to prevent the emergence of drug-resistant virus. However, G-to-A mutations caused by $A 3$ proteins in the retrovirus may cause the retrovirus to gain mutations that enable it to become drug-resistant. As shown in the study by Fourati et al., peripheral blood mononuclear cells (PBMCs) and other body fluids/tissues obtained from 30 HAART-treated patients were examined for possible drug-resistance mutations. It was found that A3-dependent hypermutations in addition to viral recombination could lead to viral evolution [6]. 
Additionally, in a study by Mulder et al., it was found that G-to-A hypermutations caused by $A 3 G$ reduces the viral infectivity and could also lead to viral diversification [11]. In this study, it was demonstrated that defective Vif mutants with suboptimal anti-A3G activity could lead to the production of proviruses resistant to the drug lamivudine (3TC) (which inhibits the retroviral reverse transcriptase) before drug exposure. Although the proviruses were not able to replicate, when they recombined with wild-type HIV-1, they produced competent viruses that were resistant to the drug 3TC [11].

In a similar study by Hernandez et al., it was found that sublethal mutations induced by $A 3 G$ could lead to viral diversification. They infected humanized mice with different variants of HIV (that were mutated via A3-independent means), and they found that the wild type HIV strain had a higher fitness compared to the mutant strains prior to its treatment with 3TC [12]. However, when they started the treatment with the drug, they found that the mutant strain was less susceptible to the antiretroviral drug's effect because of its defectiveness. In other words, because the mutant HIV strain is less fit, the drug has a decreased effect on it compared to the wild type, hence the mutant strains become resistant to the drug [12]. Thus, these studies show that sublethal mutations caused by $A 3 G$, increase viral diversification which could make the virus gain resistance to certain drugs. These mutations could also enable the virus to undergo immune escape.

\section{IMMUNE ESCAPE}

Immune escape refers to a situation where a pathogen evades detection by the host's immune system. In the case of retroviruses, this immune escape can be aided by $\mathrm{A} 3$ proteins $[1,10,13]$. As mentioned earlier, A3 proteins may sometimes cause sublethal mutations, which could help the virus to avoid detection by cytotoxic T cells (CTL), which kill infected cells $[1,10,13]$. These sublethal mutations may alter CTL epitopes or lead to mutations that alter peptide degradation and its presentation by the human leukocyte antigen (HLA) proteins on the cell surface [10, 13]. HLA proteins are human major histocompatibility complex (MHC) proteins that present peptides to CTLs, thereby allowing recognition of infected cells [10, 13]. In a study by Grant and Larijani, it was found that $A 3 G / A 3 F$ could cause mutations in HIV peptides that prevented the virus from being detected by CTLs, hence enabling it to escape the host cell's immune response [13]. Similarly, a study by Borzooee et al. showed that A3G-dependent mutations could lead to decreased binding affinity of HLA to the peptide epitopes, which also enables viral immune escape [10]. In this study, it was observed that the sequences that encode CTL epitopes in HIV are rich in A3G-preferred deamination motifs, which preferentially mutate the $\mathrm{C}$ in $\mathrm{CC} \underline{\mathrm{C}}, \mathrm{TC} \underline{\mathrm{C}}$, and ACE [10]. This could result in missense mutations that alter CTL epitopes. Thus, these mutations could help the virus evade detection by the host cell's immune response, which enables it to continue to spread in an individual.

These studies show that although A3G could result in lethal mutations in the virus, sublethal mutations could cause viral immune escape, where CTLs are no longer able to recognize infected cells to target them for killing. This could lead to natural selection and viral evolution, where the viruses are able to acquire beneficial mutations that enable them to become more fit and harmful/difficult to treat.

\section{CONCLUSION}

In conclusion, many studies have shown the effects of the cytidine deaminase activity of the A3 genes on retroviral evolution. APOBEC3 proteins are known to inhibit viral infection through their cytidine deaminase activity $[1,2$, 3 , 14]. However, lower expression of A3 proteins in cells could also lead to viral evolution [3, $5,6]$. When A3 proteins such as A3G and A3F are expressed at lower levels, they are likely to introduce missense mutations in the virus, which instead of inhibiting the virus, helps it ob- 
tain beneficial mutations that allow it to become more fit and able to evade the cells' immune response $[10,13]$ and develop drug resistance $[6$, $11,12]$. Also, many studies show that HIV can evolve to overcome A3's cytidine deaminase activity by encoding Vif's variants that more effectively target A3 for proteasomal degradation $[1,3,5,14]$. Moreover, Fourati et al. and Mulder et al., both showed that in addition to A3-dependent mutations, viruses could also evolve via recombination, where defective viruses after recombination gain drug resistance $[6,11]$.

These studies collectively suggest that A3 proteins play an important role in the host cells' immune response and help the cells to combat viral infection. Although, these studies highlight the importance of A3 proteins, they also show the limitations of its use in preventing viral infection, since HIV-1 Vif protein inhibits A3 proteins' antiviral activity. Future studies should focus on developing drugs that would inhibit HIV-1 Vif's ability to bind and degrade A3 proteins, or enable A3 proteins, specifically A3G, to overcome Vif activity, so that they could better inhibit retroviral infection and evolution.

\section{AUTHOR INFORMATION}

\section{Corresponding Author}

*Mary-Benedicta Obikili

mobiki2@uic.edu

\section{Funding Sources}

This research was supported by the $\mathrm{NIH} / \mathrm{NI}-$ AID grant R01AI085015 and the L@s GANAS project (HSI STEM grant from Dept. of Education P031C160237).

\section{Competing Interests}

The author declares no competing financial and non-financial interests.

\section{ACKNOWLEDGMENTS}

I would like to thank L@s Ganas Research Fellowship and the Chancellor's Undergraduate Research Award (CURA) for funding my research. I would also like to thank the entire Ross lab, for their support during my research. I especially want to thank Dr. Karen Salas Briceno, for all her mentorship and guidance during my research. Finally, I am especially grateful to Dr. Susan R. Ross, who provided me with immense knowledge and guidance during my time at her lab. I am truly grateful for the opportunity of doing research at the Ross lab.

\section{REFERENCES}

[1] Stavrou S, Ross SR (2015) APOBEC3 Proteins in Viral Immunity. The Journal of Immunology 195(10):4565-4570.

[2] Armitage AE, et al. (2014) Possible Footprints of APOBEC3F and/or Other APOBEC3 Deaminases, but Not APOBEC3G, on HIV-1 from Patients with Acute/Early and Chronic Infections. Journal of Virology 88(21):1288212894.

[3] Kim E-Y, et al. (2014) Human APOBEC3 Induced Mutation of Human Immunodeficiency Virus Type-1 Contributes to Adaptation and Evolution in Natural Infection. PLoS Pathogens 10(7). doi:10.1371/journal.ppat. 1004281.

[4] Sousa MM, Krokan HE, Slupphaug G (2007) DNA-uracil and human pathology. Molecular Aspects of Medicine 28(3-4):276-306.

[5] Sato K, et al. (2014) APOBEC3D and APOBEC3F Potently Promote HIV-1 Diversification and Evolution in Humanized Mouse Model. PLoS Pathogens 10(10). doi:10.1371/journal. ppat.1004453.

[6] Fourati S, et al. (2014) Differential impact of APOBEC3-driven mutagenesis on HIV evolution in diverse anatomical compartments. Aids 28(4):487-491.

[7] Parker N, Schneegurt M, Tu A-HT, Forster BM, Lister P (2016) Microbiology (OpenStax, Houston, TX). 
[8] Herron JC, Freeman S (2014) Evolutionary analysis (Pearson). 5th Ed.

[9] An P, et al. (2004) APOBEC3G genetic variants and their influence on the progression to AIDS. Journal of Virology 78: 11070-11076.

[10] Borzooee F, Joris KD, Grant MD, Larijani M (2019) APOBEC3G Regulation of the Evolutionary Race Between Adaptive Immunity and Viral Immune Escape Is Deeply Imprinted in the HIV Genome. Frontiers in Immunology 9. doi:10.3389/fimmu.2018.03032.

[11] Mulder LCF, Harari A, Simon V (2008) Cytidine deamination induced HIV-1 drug resistance. Proceedings of the National Academy of Sciences 105(14):5501-5506.

[12] Hernandez MM, et al. (2019) Impact of Suboptimal APOBEC3G Neutralization on the Emergence of HIV Drug Resistance in Humanized Mice. Journal of Virology 94(5). doi:10.1128/jvi.01543-19.

[13] Grant M, Larijani M (2017) Evasion of adaptive immunity by HIV through the action of host APOBEC3G/F enzymes. AIDS Research and Therapy 14(1). doi:10.1186/s12981-0170173-8.

[14] Cadena C, et al. (2016) The effect of HIV1 Vif polymorphisms on A3G anti-viral activity in an in vivo mouse model. Retrovirology 13(1). doi:10.1186/s12977-016-0280-y. 